\title{
INFLUENCIA DE LA MINERALOGÍA DE ARCILLAS, TEXTURA Y CONTENIDO DE CARBONO ORGÁNICO SOBRE EL ÍNDICE DE FRIABILIDAD DE SUELOS CULTIVADOS CON CAÑA DE AZÚCAR
}

\author{
Edgar Alvaro Ávilal ${ }^{1 / *}$, Aquiles Enrique Darghan ${ }^{* *}$, Fabio Rodrigo Leiva ${ }^{* *}$ \\ Palabras clave: Arcillas 2:1; arcillas 1:1; materia orgánica; resistencia al corte; fracción arena. \\ Keywords: Clay 2:1; clay 1:1; organic material; tensile strength; sand fraction.
}

Recibido: 22/10/15

Aceptado: 21/06/16

\section{RESUMEN}

La friabilidad es una propiedad física esencial en el manejo mecánico del suelo y su comportamiento está asociado a la naturaleza de otras propiedades edáficas; que requiere ser estudiada de manera integral con diferentes propiedades mineralógicas, físicas y químicas. Se evaluó el efecto del dominio de los minerales arcillosos tipo 1:1 (caolinitas) y 2:1 (esmectitas y vermiculitas), la textura y el contenido de carbono orgánico (CO), sobre el índice de friabilidad (IF) de suelos cultivados con caña de azúcar. El IF se determinó por el método del coeficiente de variación, a partir del límite inferior del intervalo de confianza $\left(\mathrm{IF}_{\mathrm{i}}\right)$. Se utilizaron 7280 agregados de los horizontes Ap y A1, tomados en 18 fincas localizadas en el Valle Geográfico del Río Cauca, Colombia. Se determinó la mineralogía de la fracción arcilla a partir de difracción de rayos $\mathrm{X}$, la distribución granulométrica por el método de la pipeta y el contenido de $\mathrm{CO}$ por el método de Walkley \& Black. El análisis estadístico se realizó a partir de un enfoque descriptivo e inferencial, univariante y multivariante. No se encontró efecto significativo de la dominancia de arcillas 1:1 y 2:1 sobre la variable respuesta $\mathrm{IF}_{\mathrm{i}}$; sin embargo, de manera descriptiva se observaron valores más altos deI $\mathrm{IF}_{\mathrm{i}}$ en suelos con dominio de arcillas 1:1. No se encontró efecto

1 Autor para correspondencia. Correo electrónico: eaavila@ut.edu.co

* Universidad del Tolima, Ibagué, Colombia.

\section{ABSTRACT}

Influence of clay mineralogy, texture, and organic carbon content on the friability index of soils cultivated with sugar cane. Friability is a physical property that is essential to the mechanical management of soil and its behavior is associated with the nature of other edaphic properties; therefore, it must be investigated with integrated studies using different mineralogical, physical, and chemical properties. The effect of the predominance of the clay mineral types $1: 1$ and $2: 1$, the texture, and the organic carbon (CO) content, on the friability index (IF) of soils cultivated with sugar cane were evaluated. The IF was determined with the coefficient of variation method, using the lower limit of the confidence interval $\left(\mathrm{IF}_{\mathrm{i}}\right)$ as reference. A total of 7280 aggregates from the Ap and A1 horizons were used, taken from 18 farms located in the Valle Geográfico of the Cauca River region, Colombia. The mineralogy of the clay fraction was determined with X-ray diffraction, the granulometric distribution by the pipette method, and the $\mathrm{CO}$ content by the Walkley \& Black method. The statistical analysis was conducted from a descriptive and inferential focus, univariate and multivariate. No significant effect from the predominance of clays 1:1 and 2:1 on the $\mathrm{IF}_{\mathrm{i}}$ response variable was found; however,

\footnotetext{
* Universidad Nacional de Colombia, Bogotá, Colombia
} 
significativo atribuible a las fracciones de arcilla y limo total (FaT y FLT) sobre el IF $_{\mathrm{i}}$ de los suelos, por el contrario, la fracción arena total (FAT) y el contenido de $\mathrm{CO}$ presentaron efecto altamente significativo en el $\mathrm{IF}_{\mathrm{i}}$, con valores más altos del $\mathrm{IF}_{\mathrm{i}}$ en los suelos con mayores contenidos de $\mathrm{CO}$ y menores contenidos de la FAT.

\section{INTRODUCCIÓN}

La friabilidad es una propiedad física asociada con la capacidad del suelo para soportar el crecimiento de la planta y para minimizar la energía requerida para la labranza (Munkholm 2011). El consumo eficiente de energía en la agricultura, especialmente en la labranza, minimiza el impacto ambiental y reduce la degradación del suelo (Sefeedpari et al. 2014). La labranza y mecanización intensiva tienen efecto negativo sobre la fragmentación del suelo y su friabilidad (Munkholm 2011). En este contexto, la cuantificación de la friabilidad a partir de la medición de la resistencia al corte (RC), definida como la fuerza por unidad de área necesaria para causar la ruptura de los agregados (Dexter y Watts 2001), aporta información para el adecuado manejo de los suelos y el mejoramiento de su calidad física (Macks et al. 1996). La RC en particular, al estar controlada por microfisuras y grietas, es altamente sensible a la microestructura del suelo (Watts y Dexter 1998). La friabilidad es una propiedad que depende de otras propiedades del suelo (Munkholm et al. 2012), entre estas, el CO, la cual influye en la resistencia al corte (Schjǿnning et al. 2011).

La arcilla y la materia orgánica (MO) son pilares de la estructura del suelo; las arcillas que floculan forman dominios estables y junto con la MO actúan como material de unión entre las partículas minerales en la formación de la higher $\mathrm{IF}_{\mathrm{i}}$ values were observed in the descriptive approach in the soils with a predominance of clays 1:1. There was no significant effect attributable to the total silt and clay fractions (FaT and FLT) on the $\mathrm{IF}_{\mathrm{i}}$ of the soils; rather, the total sand fraction (FAT) and the CO content showed highly significant effects on the $\mathrm{IF}_{\mathrm{i}}$, with higher $\mathrm{IF}_{\mathrm{i}}$ values in the soils with higher $\mathrm{CO}$ contents and lower FAT contents.

estructura (Oades 1984). La MO se puede alojar en los pequeños poros, que generan vínculos entre las partículas e influyen en la resistencia del suelo, especialmente en los agregados de mayor tamaño (Guérif 1994), sin embargo, Goldberg et al. (1990), sostienen que los compuestos orgánicos pueden ser adsorbidos sobre las arcillas que modifican la RC de los agregados del suelo.

Macks et al. (1996), sostienen que la resistencia del suelo y su friabilidad están influenciadas por el tipo y cantidad de la fracción arcilla, los cationes intercambiables y la proporción de arcilla dispersable en el suelo. Dexter et al. (2008) encontraron que la dispersibilidad de la arcilla aumenta con la cantidad de arcilla no complejada por el $\mathrm{CO}$; concluyeron que aproximadamente $10 \mathrm{~g}$ de arcilla se complejan con $1 \mathrm{~g}$ de CO. Se ha demostrado que grandes cantidades de arcilla dispersable pueden aumentar la RC y reducir el IF en agregados secos (Kay y Dexter 1992).

Las asociaciones órgano-minerales relativamente estables pueden proporcionar una mayor resistencia a la ruptura física de los agregados del suelo con la labranza, especialmente en suelos de texturas finas (Jindaluang et al. 2013). Así mismo, Bruun et al. (2010) mencionan que la capacidad de estabilización de la MO no es igual para todos los tipos de arcilla y sugieren que la capacidad es mayor en arcillas 2:1 que en las 1:1 y decrece para seguir la secuencia alófana> esmectita>illita>caolinita. 
El objetivo de esta investigación es evaluar el efecto del dominio de los minerales arcillosos tipo 1: 1 y 2:1, la textura y el contenido de carbono orgánico en el índice de friabilidad del suelo.

\section{MATERIALES Y MÉTODOS}

En los departamentos del Valle del Cauca y Cauca, se localizaron 18 fincas cultivadas en caña de azúcar en las que se identificaron suelos con dominancia de arcillas tipo 1:1 y 2:1 en su capa productiva. Los sitios fueron ubicados a partir del estudio de suelos del área cañera realizado por el IGAC (2006). Las fincas se localizaron en el bosque seco tropical (bs-T) con altitud de los predios entre los 927 y $1011 \mathrm{msnm}$, temperatura media anual entre los 22,7 y $23,5^{\circ} \mathrm{C}$ y precipitación entre 773 y $1542 \mathrm{~mm}$ anuales. El mismo estudio permitió la confirmación de la descripción de los perfiles en campo, además, se estableció como clasificación taxonómica correspondiente a los órdenes Inceptisol, Molisol, Vertisol, Alfisol y Ultisol (USDA 2010), caracterizados por estar bien a imperfectamente drenados, profundos a moderadamente profundos y con predominio de las fracciones arcilla y limo en su capa productiva (Cuadro 1). Geomorfológicamente se localizaron dentro de la planicie aluvial del río Cauca, distribuidos en terrazas bajas (Inceptisoles), medias (Molisoles y Vertisoles) y altas (Alfisoles y Ultisoles), especialmente localizados en los planos de terraza (bancos bien drenados) y unos pocos, Vertic Endoaqualfs y Typic Endoaquerts, en pequeños bajos imperfectamente drenados. Estos suelos se han originado predominantemente de sedimentos aluviales finos y medios transportados por las corrientes fluviales del sector. $\mathrm{La}$ topografía de las fincas en general presenta una pendiente dominante en el rango 1-3\%. Los contenidos de $\mathrm{CO}$ promedio ponderado por finca oscilaron entre 10,7 y 24,4 g. $\mathrm{kg}^{-1}$ en el horizonte Ap y entre 10,4 y 23,6 g. $\mathrm{kg}^{-1}$ en el horizonte A1. 


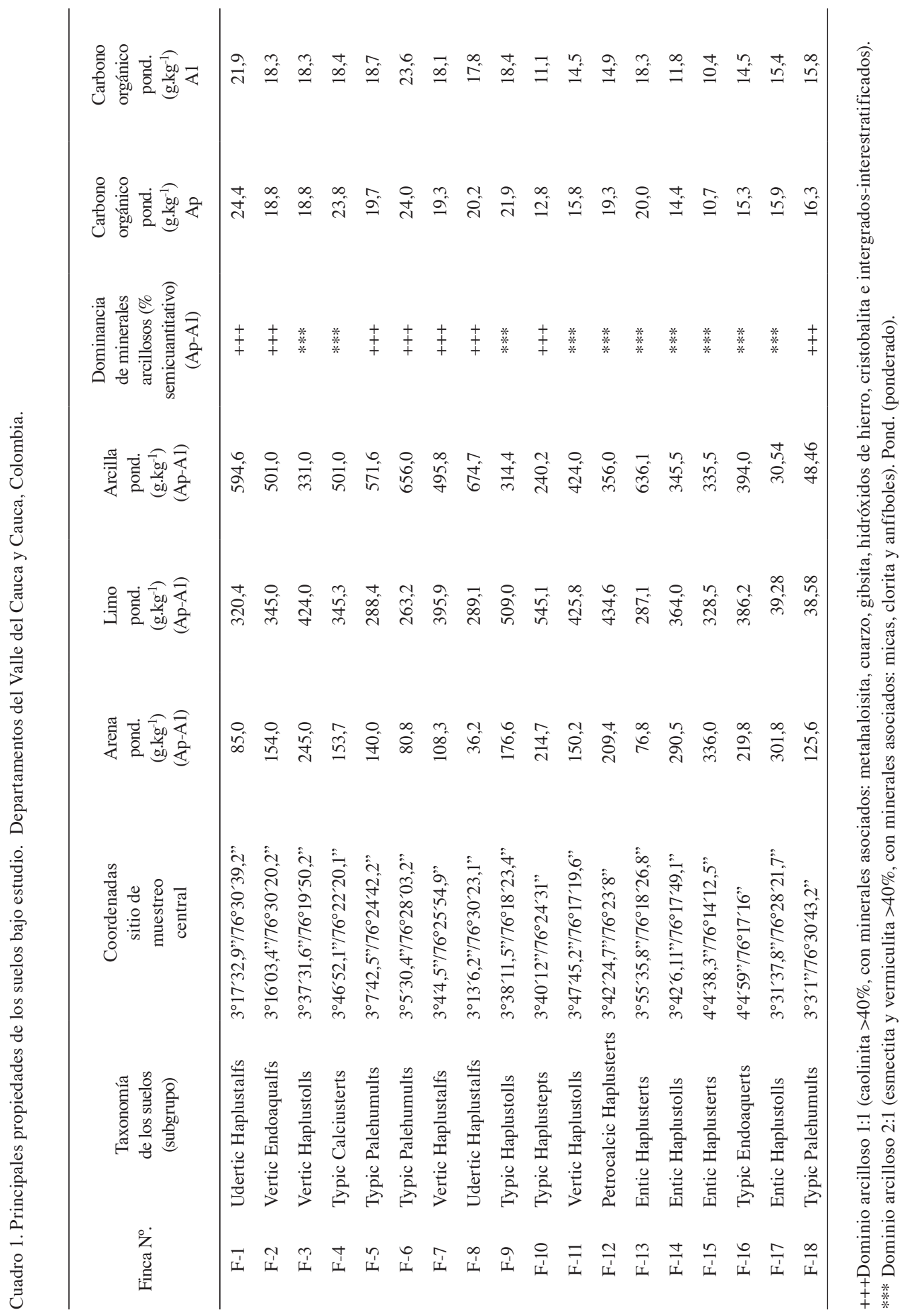


Del estudio detallado de suelos se identificó la unidad taxonómica de interés y dentro de los límites de la misma, se ubicaron 5 sitios de muestreo (excepto en la primera finca en la cual se seleccionaron 6 sitios), distantes entre sí aproximadamente $100 \mathrm{~m}$. En cada sitio se abrieron cajuelas de 50 x $50 \mathrm{~cm}$ de lado y $50 \mathrm{~cm}$ de profundidad en las que se delimitaron el horizonte superficial (Ap) y el subsiguiente (A1). De manera independiente por horizonte se realizó el muestreo de suelos que incluyó la toma de bloques de suelo $(30$ x $18 \mathrm{~cm}$ de lado y $12 \mathrm{~cm}$ de espesor aproximadamente) necesarios para la obtención de los agregados del suelo.

Se recolectaron 182 bloques de suelo (91 por horizonte), a partir de los cuales se separaron en laboratorio los agregados manualmente y así aprovechar los planos de debilidad natural del suelo. Los agregados se secaron en invernadero durante 5 días a temperatura promedio diurna de $29^{\circ} \mathrm{C}$. Posteriormente, mediante juego de tamices, se separaron los agregados por tamaño en 4 rangos que resultaron dominantes en el fraccionamiento: 2,0 a $4,7 \mathrm{~mm}$; 4,8 a 9,5 $\mathrm{mm}$; 9,6 a 18,9 $\mathrm{mm}$ y $19,0-35,7 \mathrm{~mm}$. Se seleccionaron 10 agregados por rango, a partir de la metodología de Guimaraes et al. (2009), para lo cual se colocaron para secado final a $40^{\circ} \mathrm{C}$ durante 48 horas. Las muestras se almacenaron en recipientes herméticos para evitar ganancia de humedad ambiental hasta la prueba de compresión.

Se obtuvieron 7280 agregados, 40 por horizonte muestreado, que se pesaron individualmente y se sometieron al ensayo de compresión en un equipo CBR (Soiltest - CF410) de 2 velocidades, integrado por un par de platos paralelos entre los cuales los agregados fueron comprimidos. En cada ensayo se aplicó esfuerzo a una tasa de deformación constante de $0,07 \mathrm{~mm} \cdot \mathrm{s}^{-1}$ (Watts y Dexter 1998). La RC se obtuvo de la ecuación descrita por Dexter y Kroesbergen (1985), a saber, $\mathrm{RC}=0,576\left(\mathrm{P} / \mathrm{D}_{\mathrm{e}}{ }^{2}\right)$, donde $\mathrm{RC}$ es la resistencia al corte $(\mathrm{kPa}), \mathrm{P}$ representa el pico de fuerza al fallar el agregado, y $\mathrm{D}_{\mathrm{e}}$ el diámetro efectivo por agregado ( $\mathrm{mm})$.

El IF se determinó a partir del límite inferior del coeficiente de variación, denotado ahora con $\mathrm{IF}_{\mathrm{i}}$ propuesto por Dexter y Watts (2001), es decir, $\left\lfloor F_{i}=\frac{S_{\bar{r}}}{y}-\frac{S_{y}}{y \sqrt{2 n}}\right\rfloor$

Donde Sy es la desviación estándar muestral de los valores medidos de $\mathrm{RC}(\mathrm{kPa}), \overline{\mathrm{y}}$ es la media de la medidas de RC y, n, el número de repeticiones, que en este caso se correspondió a 10 agregados. Se tomó el límite inferior como resultado del enfoque multivariante propuesto por Avila et al. (2015), ya que la medida univariante en este límite es más próxima al valor obtenido mediante el coeficiente de variación multivariante, para el cual solo se obtuvo una estimación puntual.

Para el fraccionamiento de partículas minerales de tamaño inferior a $2 \mathrm{~mm}$, inicialmente se llevó a cabo la eliminación de agentes cementantes naturales del suelo (método de la pipeta) (IGAC 2006a). Posteriormente, mediante procesos de dispersión y tamizado en húmedo se aislaron las fracciones arena muy gruesa (2-1 $\mathrm{mm})$, arena gruesa $(1-0,5 \mathrm{~mm})$, arena media $(0,5-0,25)$, arena fina $(0,25-0,1 \mathrm{~mm}) \mathrm{y}$ arena muy fina (0,1-0,05 mm). Según Barré et al. (2014), la fracción arcilla normalmente se extrae por sedimentación o centrifugación; en este trabajo, las fracciones limo grueso $(0,05-0,02 \mathrm{~mm})$, limo fino $(0,02-0,002 \mathrm{~mm})$ y arcilla $(<0,002 \mathrm{~mm})$ se obtuvieron a partir de dispersión, agitación, sedimentación y toma de alícuotas en diferentes periodos de tiempo. En los suelos hubo predominio de texturas arcillosa, franco arcillosa y arcillo limosa (Figura 1). 

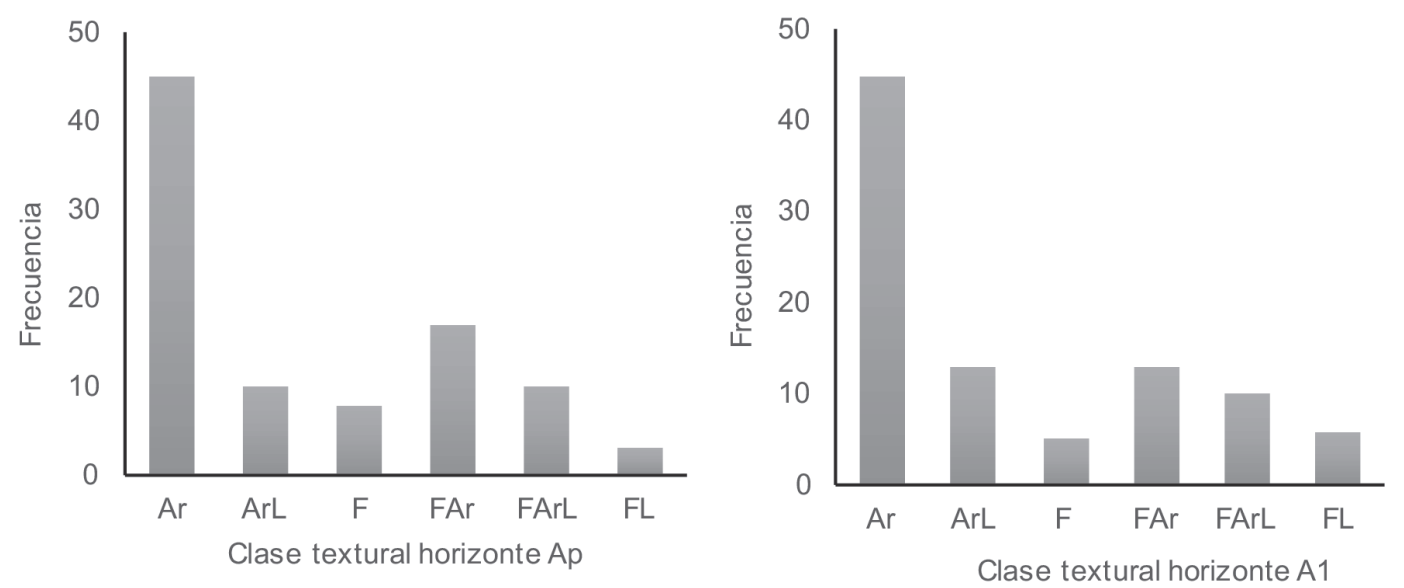

Fig. 1. Distribución de las clases texturales de los horizontes Ap y A1. Departamentos del Valle del Cauca y Cauca, Colombia.

Ar: arcillosa, ArL: arcillo limosa, F: franca, FAr: franco arcillosa, FArL: franco arcillo limosa y FL: franco limosa.

La fracción de arcilla aislada en las 182 muestras se sometieron 4 tratamientos con cationes en solución previo a su análisis con el equipo de difracción de rayos X (DRX): i) solución de arcilla saturada con $\mathrm{MgCl}_{2} 1 \mathrm{~N}$; ii) solución de arcilla saturada con $\mathrm{MgCl}_{2} 1 \mathrm{~N}$ y $\mathrm{C}_{2} \mathrm{H}_{6} \mathrm{O}_{2}$; iii) solución de arcilla saturada con $\mathrm{KCl} 1 \mathrm{~N}$; y iv) solución de arcilla saturada con $\mathrm{KCl} 1 \mathrm{~N}$, seca al aire y posteriormente calentada a $550^{\circ} \mathrm{C}$. Esta técnica de identificación (IGAC 2006a) utiliza los tratamientos mencionados para identificar mediante la difracción de rayos $\mathrm{X}$, el comportamiento diferencial de los minerales de la fracción arcilla a partir de la saturación con los diferentes cationes (magnesio y potasio), las moléculas de etilenglicol y el calentamiento a $550^{\circ} \mathrm{C}$. El proceso de DRX para cada muestra se realizó durante un tiempo aproximado de 7 minutos en un equipo Panalytical Empyrean serie 2 - 2010, con velocidad de barrido $2^{\circ} \mathrm{T}$ x minuto. El barrido de cada muestra se realizó entre los ángulos $2^{\circ}$ y $40^{\circ} \mathrm{T}$. En relación con el $\mathrm{CO}$, este se determinó mediante el procedimiento de digestión vía húmeda de Walkley \& Black (IGAC 2006a).
Inicialmente se realizó un análisis de componentes principales (ACP) para caracterizar la dominancia mineral presente en la fracción arcilla. El criterio de extracción de componentes se basó en el diagrama de sedimentación (efecto codo). Se identificó el dominio de minerales arcillosos tipo 1:1 y 2:1 y se construyó el diagrama tridimensional de los pesos de los 3 primeros componentes extraídos para representar la dominancia del tipo de arcilla en el biplot. A partir del componente descriptivo se generaron las cargas de la covariable asociada a la dominancia (variables originales: 11 minerales de la fracción arci1la) y luego se realizó un análisis de covarianza con el SAS (Statistical Analysis System, v. 9.3) para un diseño anidado en 3 etapas, con las covariables CO y FAT, pues las covariables FaT, FLT y los diferentes tamaños de la fracción arena, no cumplieron un supuesto necesario para permanecer en el modelo, a saber, relación lineal con la respuesta evaluada. La variable respuesta fue el $\mathrm{IF}_{\mathrm{i}}$, por lo que se generaron 36 combinaciones de niveles de los factores tamaño de agregado, tipo de horizonte y textura. Se calcularon estadísticas descriptivas (media y coeficientes de variación) 
para el $\mathrm{IF}_{\mathrm{i}}$, los promedios de $\mathrm{CO}$ y la FAT, cada uno de ellos por tamaño de agregado ( 2 a 9,5; 9,6 a 18,9 y 19 a $35,7 \mathrm{~mm}$ ), horizonte morfogenético (Ap y A1) y textura: arcillosa (Ar), arcillo limosa (ArL), franca $(\mathrm{F})$, franco arcillosa (FAr), franco arcillo limosa (FArL) y franco limosa (FL).

\section{RESULTADOS Y DISCUSIÓN}

Algunos estudios previos que han relacionado la influencia de minerales de arcilla tipo 1:1 y 2:1 con la RC fueron trabajados con agregados remoldeados (Barzegar et al. 1995). En esta investigación se puso a prueba el efecto de la dominancia de estos minerales en el $\mathrm{IF}_{\mathrm{i}}$ de agregados naturales del suelo. La Figura 2 presenta los 3 componentes extraídos del Análisis de Componentes Principales, los cuales explicaron más del $63 \%$ de la variabilidad encontrada en los datos. En la dirección del primer componente se encontró la información de mayor interés para la segregación de la dominancia del tipo de arcillas 1:1 o 2:1. Los valores con pesos positivos se asociaron a los minerales esmectita(em), vermiculita(vr), micas(mi), clorita (cl) y anfíboles (af), los cuales se agruparon en el conjunto de arcillas del tipo 2:1; mientras que los pesos negativos para este componente se asociaron a los minerales caolinita (ca), metahaloisita (mt), cuarzo (qz), gibsita (gb), hidróxidos de hierro (hf), cristobalita (cr) e intergrados-interestratificados (ii), conforman el dominio de arcillas tipo 1:1. Estas cargas se usaron como covariable para el análisis de covarianza, ya que las 2 dominancias quedaron bien representadas en el primer componente.

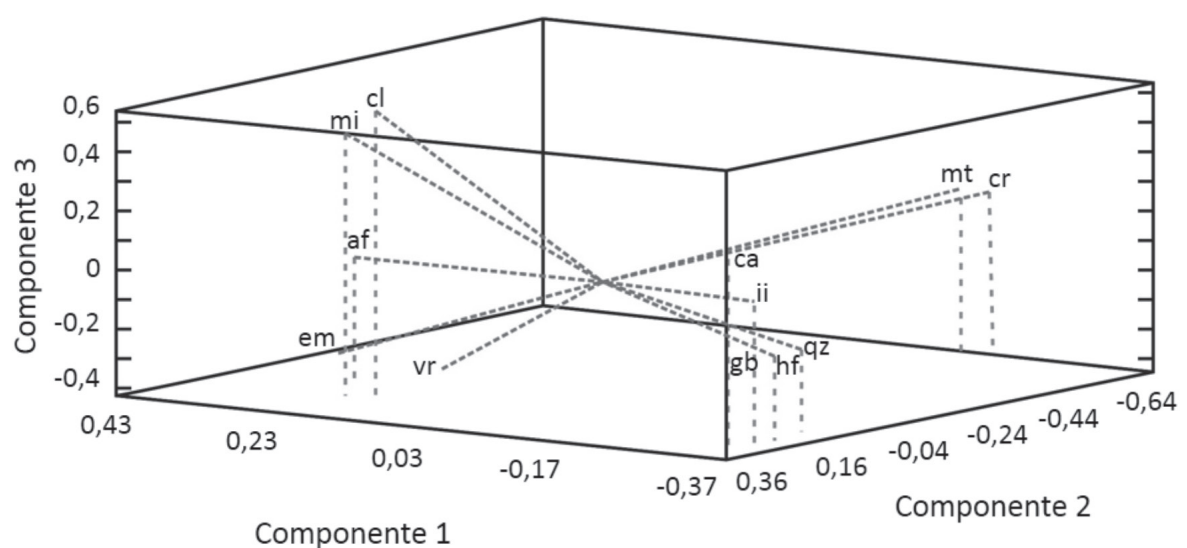

Fig. 2. Diagrama tridimensional de los pesos de los componentes extraídos del análisis de componentes principales para las variables minerales de la fracción arcilla. Departamentos del Valle del Cauca y Cauca, Colombia.

En el Cuadro 2 se resume el análisis de covarianza en la que se observa como el efecto de la dominancia de arcillas 1:1 y 2:1 asociada a las cargas del componente extraído no resultó significativo $(\mathrm{p}>5 \%)$. En este cuadro se encuentran también los resultados del análisis en un modelo reducido con aquellas variables de efectos no nulos desde el punto de vista estadístico, en el que se encontró que las covariables $\mathrm{CO}$ y FAT resultaron altamente significativas en el ajuste del modelo $(\mathrm{p}<1 \%)$, al igual que el tamaño de los agregados anidado en el efecto conjunto del horizonte y la textura $(\mathrm{p}<1 \%)$. La interacción que involucró el tamaño agregado anidada en la interacción (horizonte*textura) resultó significativa, por lo que no se realizaron las pruebas de 
Cuadro 2. Análisis de covarianza para el diseño anidado en 3 etapas. Departamentos del Valle del Cauca y Cauca, Colombia.

\begin{tabular}{|c|c|c|c|c|c|}
\hline Fuente & $\mathrm{gl}$ & Suma de cuadrados & cuadrado medio & Cociente F & $\operatorname{Pr}>\mathrm{F}$ \\
\hline Horizonte morfogenético & 1 & 0,2768 & 0,2768 & 24,10 & $<0,0001$ \\
\hline Textura (horizonte) & 10 & 0,2880 & 0,0288 & 2,51 & 0,0060 \\
\hline Tamaño agregado (horizonte*Textura) & 24 & 0,8433 & 0,0351 & 3,06 & $<0,0001$ \\
\hline FAT & 1 & 0,1526 & 0,1526 & 13,29 & 0,0003 \\
\hline $\mathrm{CO}$ & 1 & 0,0898 & 0,0898 & 7,82 & 0,0054 \\
\hline Dominancia de arcillas $1: 1$ y $2: 1$ & 1 & 0,0015 & 0,0015 & 0,13 & 0,7136 \\
\hline Error & 504 & 5,7898 & 0,0114 & & \\
\hline Total & 542 & 7,6015 & & & \\
\hline
\end{tabular}

comparaciones de medias para efectos principales, sin embargo, se realizaron los promedios ajustados por mínimos cuadrados para las variables involucradas en el modelo, así como los promedios observados del $\mathrm{IF}_{\mathrm{i}}$ para la dominancia de las arcillas, pues este aspecto se consideró relevante en este trabajo aun sin encontrar un efecto no significativo en el modelo ajustado. Además, el despliegue gráfico permitió entender la naturaleza de la interacción y permitió ver la relación de las variables explicativas con la respuesta, a saber, el índice de friabilidad en su límite inferior.

El Cuadro 2 solo presenta algunos efectos (se evidencia en los grados de libertad), específicamente los que resultaron significativos, con excepción del tipo de dominancia, pues la esencia de la presente investigación se radicó en este efecto. Los paréntesis en los efectos de la fuente de variación solo indican una estructura de anidamiento de factores.

Aunque el efecto de la dominancia de arcillas no mostró significancia (Cuadro 2), resultó de interés destacar cómo de manera descriptiva (Figura 3), los perfiles mostraron que a medida que se hizo mayor el tamaño del agregado, mayor fue también el $\mathrm{IF}_{\mathrm{i}}$ en cada una de las dominancias de arcilla; a este respecto y de acuerdo con Schjǿnning et al. (2011), en general, la RC de los agregados naturales de suelos bien manejados, disminuye al aumentar el tamaño de agregado y se incrementa la friabilidad. El $\mathrm{IF}_{\mathrm{i}}$ de los suelos con dominio de arcillas 1:1 fue superior en todos los tamaños, lo cual coincide con lo reportado por Barzegar et al. (1995), en Alfisoles y Vertisoles Australianos, en los que las arcillas esmectíticas, con alta capacidad de intercambio catiónico y tamaño fino $(<0,02 \mu \mathrm{m})$, presentaron una mayor resistencia que aquellos con dominio de caolinitas e illitas, lo cual en términos de friabilidad, indica una mayor friabilidad en suelos con dominio de arcillas 1:1 con respecto a los de dominio $2: 1$. Se encontró también que en los agregados de mayor tamaño (a partir del rango entre 9,6 y 18,9 $\mathrm{mm}$ ), el valor del $\mathrm{IF}_{\mathrm{i}}$ de los 2 dominios arcillosos mostró la mayor diferencia. De acuerdo con Guérif (1994), la MO puede disponerse en el espacio poroso de menor tamaño que fortalece el vínculo entre las partículas minerales, que llevan al incremento de su RC y por otra parte a la disminución de su friabilidad, especialmente en los agregados de mayor tamaño. En la presente investigación, este postulado explica en parte los valores más bajos del $\mathrm{IF}_{\mathrm{i}}$ en los suelos con dominio de arcillas tipo 2:1 (especialmente en los agregados grandes). Así mismo, la alta carga de las arcillas esmectiticas relacionada con una mayor superficie de unión, tanto con otras partículas minerales como con la MO, confiere a los suelos con dominio de 


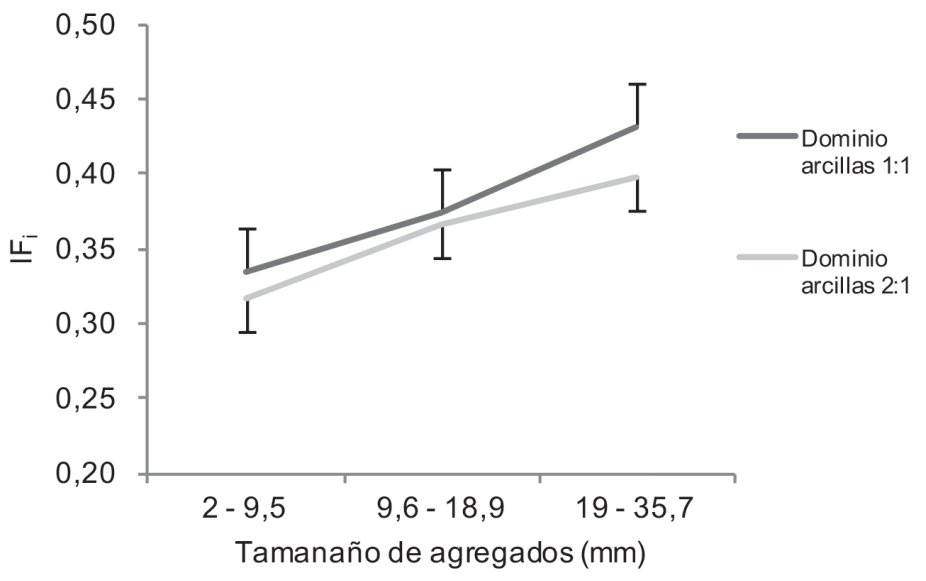

Fig. 3. Perfiles del IFi por tipo de dominio arcilloso y tamaño de los agregados. Departamentos del Valle del Cauca y Cauca, Colombia. (Las barras en cada perfil indican el error estándar de la media del IFi; uno positivo y uno negativo solo para evitar el solapamiento de las barras).

este tipo de arcillas una mayor resistencia por el mayor volumen de contacto entre las partículas, por la mayor capacidad de intercambio catiónico (Barzegar et al. (1995) y por la mayor capacidad de estabilización de la MO reportado en estas arcillas por Bruun et al. (2010).

Aunque no se encontró efecto significativo de la dominancia de arcillas tipo 1:1 y 2:1 en el $\mathrm{IF}_{\mathrm{i}}$, otros investigadores han demostrado la influencia de algunos óxidos en la friabilidad del suelo. En su trabajo experimental con Oxisoles del trópico, Imhoff et al. (2002) encontraron que óxidos de $\mathrm{Fe}$ y $\mathrm{Si}$ correlacionaron negativamente con la friabilidad, mientras que por el contrario la relación con formas cristalinas de Fe fue positiva. La relación negativa con los óxidos de Fe y Si fue explicada a partir de la acción cementante de estos constituyentes, pues se consideró que altos contenidos de estas formas minerales incrementaban la resistencia del suelo y reducían la friabilidad.

En relación con la textura, la influencia de esta propiedad en la friabilidad del suelo no ha sido aún bien establecida. En este sentido, Macks et al. (1996) reportaron una pobre correlación entre textura y friabilidad en Alfisoles y Oxisoles Australianos, lo cual fue atribuido a la heterogeneidad textural de los suelos estudiados. Contrario a esto, Imhoff et al. (2002) no encontró alguna correlación entre estas 2 variables cuando se trabajó con Oxisoles del trópico. En la presente investigación, se relacionó el $\mathrm{IF}_{\mathrm{i}}$ con los contenidos de arcilla, limo y arena del suelo y se encontró que en el caso de las fracciones arcilla y limo total, no hubo ningún efecto sobre los valores del $\mathrm{IF}_{\mathrm{i}}$. Lo anterior, de acuerdo con Barzegar et al. (1995), puede atribuirse al hecho de que aunque las arcillas están implicadas en la unión o cementación de las partículas del suelo, cuando son floculadas y agregadas por los iones de calcio y MO, esta función de unión o cementación se ve ampliamente reducida y la FaT puede no reflejar la resistencia y por consiguiente, la friabilidad del suelo. Contrario a lo observado con la FaT y la FLT en la presente investigación, la FAT mostró un efecto significativo sobre el $\mathrm{IF}_{\mathrm{i}}$ en el modelo estadístico ajustado (Cuadro 2). El promedio general de esta fracción presentó un porcentaje ligeramente superior en el horizonte Ap (17,55\%), en relación con el horizonte A1 (16,35\%).

En general, los suelos de textura FL presentaron los valores más altos del $\mathrm{IF}_{\mathrm{i}}$ en todos los tamaños de agregados, tanto en Ap, como en A1 (Cuadro 3), diferencia relevante en relación con 
Cuadro 3. Distribución de los promedios (celda superior) y el coeficiente de variación (celda inferior) del $\mathrm{IF}_{\mathrm{i}}$ por horizonte, textura y tamaño del agregado. Departamentos del Valle del Cauca y Cauca, Colombia.

\begin{tabular}{|c|c|c|c|c|c|c|c|}
\hline \multirow{2}{*}{ Tamaño agregados } & \multirow{2}{*}{ Horizonte } & \multicolumn{6}{|c|}{ Textura } \\
\hline & & Ar & ArL & $\mathrm{F}$ & FAr & FArL & FL \\
\hline \multirow{2}{*}{2 a $9,5 \mathrm{~mm}$} & Ap & $\begin{array}{c}0,36 \\
(29,00)\end{array}$ & $\begin{array}{c}0,33 \\
(23,05)\end{array}$ & $\begin{array}{c}0,38 \\
(29,44)\end{array}$ & $\begin{array}{c}0,33 \\
(31,07)\end{array}$ & $\begin{array}{c}0,36 \\
(18,45)\end{array}$ & $\begin{array}{c}0,45 \\
(22,61)\end{array}$ \\
\hline & $\mathrm{A} 1$ & $\begin{array}{c}0,30 \\
(23,62) \\
\end{array}$ & $\begin{array}{c}0,31 \\
(20,81) \\
\end{array}$ & $\begin{array}{c}0,29 \\
(9,60) \\
\end{array}$ & $\begin{array}{c}0,28 \\
(25,25) \\
\end{array}$ & $\begin{array}{c}0,28 \\
(39,34)\end{array}$ & $\begin{array}{c}0,35 \\
(26,87) \\
\end{array}$ \\
\hline \multirow{2}{*}{9,6 a $18,9 \mathrm{~mm}$} & Ap & $\begin{array}{c}0,41 \\
(29,29)\end{array}$ & $\begin{array}{c}0,37 \\
(33,70)\end{array}$ & $\begin{array}{c}0,37 \\
(24,56)\end{array}$ & $\begin{array}{c}0,36 \\
(26,86)\end{array}$ & $\begin{array}{c}0,38 \\
(24,50)\end{array}$ & $\begin{array}{c}0,48 \\
(9,62)\end{array}$ \\
\hline & $\mathrm{A} 1$ & $\begin{array}{c}0,35 \\
(35,30)\end{array}$ & $\begin{array}{c}0,35 \\
(30,33)\end{array}$ & $\begin{array}{c}0,35 \\
(31,81)\end{array}$ & $\begin{array}{c}0,34 \\
(15,49) \\
\end{array}$ & $\begin{array}{c}0,34 \\
(24,61)\end{array}$ & $\begin{array}{c}0,37 \\
(39,50)\end{array}$ \\
\hline \multirow{2}{*}{19 a $35,7 \mathrm{~mm}$} & Ap & $\begin{array}{c}0,40 \\
(29,75)\end{array}$ & $\begin{array}{c}0,50 \\
(30,90)\end{array}$ & $\begin{array}{c}0,42 \\
(30,18)\end{array}$ & $\begin{array}{c}0,38 \\
(35,96)\end{array}$ & $\begin{array}{c}0,45 \\
(32,31)\end{array}$ & $\begin{array}{c}0,61 \\
(23,16)\end{array}$ \\
\hline & $\mathrm{A} 1$ & $\begin{array}{c}0,40 \\
(30,29)\end{array}$ & $\begin{array}{c}0,38 \\
(41,60)\end{array}$ & $\begin{array}{c}0,29 \\
(18,06)\end{array}$ & $\begin{array}{c}0,39 \\
(27,47)\end{array}$ & $\begin{array}{c}0,37 \\
(19,61)\end{array}$ & $\begin{array}{c}0,42 \\
(37,55)\end{array}$ \\
\hline
\end{tabular}

Ar: arcillosa, ArL: arcillo limosa, F: franca, FAr: franco arcillosa, FArL: franco arcillo limosa, FL: franco limosa.

las otras clases texturales, en el horizonte Ap. Sin embargo, al presentar altos valores del $\mathrm{IF}_{\mathrm{i}}$ tanto en los agregados pequeños como en los grandes los hace suelos de baja condición de friabilidad, ya que los pequeños agregados, por su baja resistencia son altamente susceptibles a la degradación estructural, a pesar de ser sometidos a labranza reducida. Por el contrario, los suelos con texturas FArL y ArL presentaron mejor condición de friabilidad, ya que los agregados pequeños registraron valores bajos del $\mathrm{IF}_{\mathrm{i}}$ (mayor resistencia) y los agregados grandes valores más altos del $\mathrm{IF}_{\mathrm{i}}$ (menor resistencia), por lo que se facilita la fragmentación de grandes terrones o unidades estructurales en pequeños agregados óptimos para la siembra y resistentes a la degradación.

Por otra parte, en relación con la MO, cuyo papel en la agregación ha sido ampliamente estudiado, no se ha comprobado aun un efecto claro de esta variable en el IF de los suelos. De acuerdo con lo expresado por Jindaluang et al. (2013), sostienen que una mayor RC puede ser asociada con compuestos órgano-minerales con cierto grado de estabilidad, se considera necesario analizar el CO del suelo en el contexto de las fracciones minerales que lo acompañan. En el Cuadro 4 se observa la distribución del promedio de 2 variables que mostraron efectos altamente significativos sobre el $\mathrm{IF}_{\mathrm{i}}$ en la presente investigación: el CO (valor superior en la celda del cuadro) y la FAT (valor inferior). Si bien en el horizonte Ap se encontraron los mayores valores de $\mathrm{CO}$, en ambos horizontes los contenidos más altos estuvieron asociados a las texturas Ar, ArL y FArL, con valores alrededor de entre 17 y 20 g.kg-1. Los valores más bajos de $\mathrm{CO}$ pertenecieron en ambos horizontes a los suelos con texturas F y FL, con valores próximos a 12 g.kg-1 . Lo anterior parece tener explicación en la mayor capacidad de estabilización de la MO por parte de la fracción arcilla, (Christensen 1992, Jindaluang et al. 2013), tal y como se encontró en este estudio donde los mayores contenidos de CO están asociados a la fracción más fina. Así mismo, en lo que respecta a la FAT, los valores superiores se correspondieron con los valores más bajos de $\mathrm{CO}$, que fueron para los suelos francos (F) y franco limosos (FL). 
Cuadro 4. Distribución de los promedios de CO $\left(\mathrm{g}^{\mathrm{kg}} \mathrm{kg}^{-1}\right)$ (valor superior) y FAT (\%) (valor inferior) por horizonte y textura. Departamentos del Valle del Cauca y Cauca, Colombia.

\begin{tabular}{ccccccc}
\hline \multirow{2}{*}{ Horizonte } & \multicolumn{5}{c}{ Textura } \\
& Ar & ArL & F & FAr & FArL & FL \\
\hline \multirow{2}{*}{ Ap } & 20,00 & 20,54 & 12,39 & 15,50 & 19,95 & 12,58 \\
& 11,90 & 12,43 & 36,89 & 28,10 & 14,76 & 21,93 \\
\hline \multirow{2}{*}{ A1 } & 18,64 & 17,20 & 11,10 & 13,56 & 17,76 & 12,44 \\
& 11,69 & 11,40 & 36,48 & 27,06 & 16,45 & 17,94 \\
\hline
\end{tabular}

La Figura 4 complementa la descripción tabular dada hasta el momento, en ella se representa la superficie generada por las variables $\mathrm{CO}$, FAT e IF . Es claro que a mayor FAT se encuentran los valores más bajos de CO para todo el rango del $\mathrm{IF}_{\mathrm{i}}$, mientras que la mayor friabilidad $\left(\mathrm{IF}_{\mathrm{i}}>0,5\right)$ corresponde a los más altos contenidos de $\mathrm{CO}\left(>22 \mathrm{~g} \cdot \mathrm{kg}^{-1}\right)$ y a los valores más bajos de la FAT $(<10 \%)$.

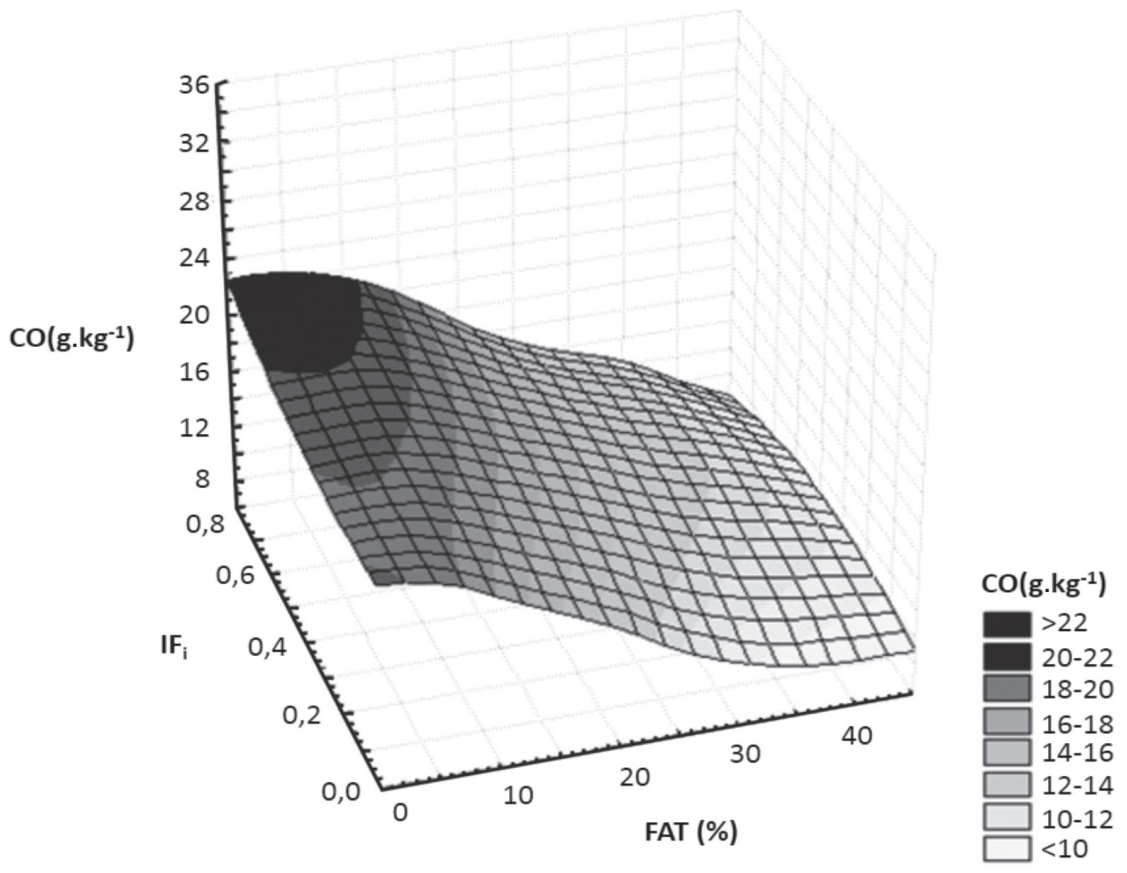

Fig. 4. Superficie generada por el método Spline para las variables carbono orgánico (CO), fracción arena total (FAT) e índice de friabilidad en su límite inferior $\left(\mathrm{IF}_{\mathrm{i}}\right)$. Departamentos del Valle del Cauca y Cauca, Colombia.

En general, los 3 tamaños de agregados presentaron mayores valores del $\mathrm{IF}_{\mathrm{i}}$ en el horizonte Ap, donde los contenidos de $\mathrm{CO}$, en su promedio general, fueron superiores en cerca de 2 g. $\mathrm{kg}^{-1}$ y el FAT en aproximadamente 1,2\% en relación con el horizonte A1 (Cuadro 5). 
Cuadro 5. Promedios generales de los contenidos de FaT, FAT y CO de los horizontes Ap y A1. Departamentos del Valle del Cauca y Cauca, Colombia.

\begin{tabular}{|c|c|c|c|c|c|c|}
\hline \multirow{2}{*}{ Horizonte } & \multicolumn{3}{|c|}{$\mathrm{IF}_{\mathrm{i}}$ para los diferentes tamaños de agregados } & \multirow{2}{*}{$\begin{array}{l}\mathrm{FaT} \\
(\%)\end{array}$} & \multirow{2}{*}{$\begin{array}{l}\text { FAT } \\
(\%)\end{array}$} & \multirow{2}{*}{$\begin{array}{c}\mathrm{CO} \\
\left(\mathrm{g} \cdot \mathrm{kg}^{-1}\right)\end{array}$} \\
\hline & 2 a $9,5 \mathrm{~mm}$ & 9,6 a $18,9 \mathrm{~mm}$ & 19 a $35,7 \mathrm{~mm}$ & & & \\
\hline Ap & 0,35 & 0,39 & 0,44 & 45,84 & 17,55 & 18,45 \\
\hline $\mathrm{A} 1$ & 0,30 & 0,35 & 0,39 & 45,53 & 16,35 & 16,72 \\
\hline
\end{tabular}

Allí mismo se observa como los promedios generales de los porcentajes de arcilla (FaT) en los horizontes Ap y A1 estuvieron muy cercanos.

A partir de esta evidencia, se considera que, probablemente, el bajo rango de variación del CO de los suelos estudiados (rango dominante sin valores extremos: $9-28$ g. $\mathrm{kg}^{-1}$ ) no permitió observar mejor la tendencia de incremento del $\mathrm{IF}_{\mathrm{i}}$ con el mayor contenido de CO. Correlaciones positivas entre estas 2 variables fueron también reportadas por Macks et al. (1996) en Alfisoles y Oxisoles, cuyos contenidos de CO oscilaron entre 7 y 61 g. $\mathrm{kg}^{-1}$, así mismo, Watts y Dexter (1998), en suelos con contenidos de CO entre 11 y 59 g. $\mathrm{kg}^{-1}$, encontraron incrementos notables de la friabilidad con los mayores contenidos de MO. Sin embrago, en otros trabajos como el realizado por Imhoff et al. (2002) en oxisoles del trópico no se demostró correlación entre estas 2 variables, lo cual fue atribuido al bajo rango de contenido de $\mathrm{CO}$ de los suelos (20 a 44 g. $\mathrm{kg}^{-1}$ ); de igual forma, Tormena et al. (2008), tampoco encontraron efecto del $\mathrm{CO}$ en el $\mathrm{IF}_{\mathrm{m}}$ en su trabajo experimental con cultivos en plantación directa y escarificación periódica en Oxisoles a $25 \mathrm{~cm}$ de profundidad. En el área de estudio, el rango de $\mathrm{CO}$ fue en general superior en el horizonte Ap $\left(8,61\right.$ - 34,01 g. $\left.\mathrm{kg}^{-1}\right)$, comparativamente con el del horizonte A1 donde el CO varió entre 8,24 y 30,55 g. $\mathrm{kg}^{-1}$; sin embargo, estos contenidos estarían dentro de un rango de variación considerado bajo, lo que de acuerdo con Imhoff et al. (2002), dificulta encontrar una clara correlación con el IF.

\section{AGRADECIMIENTOS}

A la Universidad del Tolima, a COLCIENCIAS y a la Dirección de Investigaciones de la Universidad Nacional de Colombia por el apoyo económico; a los agricultores que permitieron en sus predios el muestreo de suelos, y al Instituto Geográfico Agustín Codazzi por la colaboración en la fase analítica de la investigación.

\section{LITERATURA CITADA}

Avila, E; Leiva, F; Darghan, E; Madriñán, R. 2015. Effect of aggregate size and superficial horizon differentiation on the friability index of soils cultivated with sugar cane: a multivariate approach. Agronomía Colombiana 33(1):92-98.

Barré, P; Fernández, O; Virto, I; Velde, B; Chenu, C. 2014. Impact of phyllosilicate mineralogy on organic carbon stabilization in soils: incomplete knowledge and exciting prospects. Geoderma (235):382-395.

Barzegar, A; Oades, J; Rengasamy, P; Murray, R. 1995. Tensile strength of dry, remoulded soils as affected by properties of the clay fraction. Geoderma (65):93-108.

Bruun, T; Elberling, B; Christensen, B. 2010. Lability of soil organic carbon in tropical soils with different clay minerals. Soil Biology and Biochemistry (42):888-895.

Christensen, B. 1992. Physical fractionation of soil and organic matter in primary particle size and density separates. Advances in Soil Sciences (20):1-90.

Dexter, A; Kroesbergen, B.1985. Methodology for determination of tensile strength of soil aggregates. Journal Agriculture Engeniering Research (31):139-147.

Dexter, A; Richard, G; Arrouays, D; Czyz, E; Jolivet, C; Duval, O. 2008. Complexed organic matter controls soil physical properties. Geoderma (144):620-627.

Dexter, A; Watts, C. 2001. Tensile strength and friability. In Smith, KA; Mullins, CE. (eds.). Soil and 
Environmental Analysis: Physical Methods. 2 ed. Marcel Dekker Inc, New York, USA. p. 405-433.

Goldberg, S; Kapoor, B; Rhoades, J. 1990. Effect of aluminum and iron oxides and organic matter on flocculation and dispersion of arid zone soils. Soil Science (150):588- 593.

Guérif, J. 1994. Effects of compaction on soil strength parameters. Elsevier Science (Chapter 9):191-213.

Guimarães, R; Tormena, C; Alves, S; Fidalski, J; Blainski, E. 2009. Tensile strength, friability and organic carbon in an oxisol under a crop-livestock system. Scientia Agricola (Piracicaba, Brasil.) 66(4):499-505.

IGAC (Instituto Geográfico Agustín Codazzi, Colombia). 2006. Estudio Detallado de Suelos y Capacidad de Uso de las Tierras Sembradas con Caña de Azúcar en el Valle Geográfico del Río Cauca. Bogotá, Colombia. 483 p.

IGAC (Instituto Geográfico Agustín Codazzi, Colombia). 2006a. Métodos analíticos del laboratorio de suelos. Sexta edición. Bogotá, Colombia. 674 p.

Imhoff, S; Da Silva, P; Dexter, A. 2002. Factors contributing to the tensile strength and friability of Oxisols. Soil Science Society of America Journal (66):1656-1661.

Jindaluang, W; Kheoruenromne, I; Suddhiprakarn, A; Singh, B. 2013. Influence of soil texture and mineralogy on organic matter content and composition in physically separated fractions soils of Thailand. Geoderma (195):207-219.

Kay, B; Dexter, A. 1992. The influence of dispersible clay and wetting/drying cycles on the tensile strength of a red-brown earth. Australian Journal of Soil Research (30):297-310.

Macks, S; Murphy, B; Cresswell, H; Koen, T. 1996. Soil friability in relation to management history and suitability for direct drilling. Australian Journal of Soil Research (34):343-360.
Munkholm, L. 2011. Soil friability: A review of the concept, assessment and effects of soil properties and management (Review). Geoderma (167):236-246.

Munkholm, L; Heck, R; Deen, B. 2012. Soil pore characteristics assessed from X-ray micro-CT derived images and correlations to soil friability. Geoderma (181):22-29.

Oades, J. 1984. Soil organic matter and structural stability: mechanisms and implications for management. Plant and Soil (76):319-337.

SAS (Statistical Analysis System, USA). 2009. SAS user's guide; SAS/STAT. SAS Campus Drive, Cary North Carolina Rv. 27513. p. 206.

Schjǿnning, P; De Jonge, L; Munkholm, L; Moldrup, P; Christensen, B; Olesen, J. 2011. Clay dispersibility and soil friability-testing the soil clay-to-carbon saturation concept. Soil Science Society of America Journal. Special section: Soil Architecture and Function: 174-187.

Sefeedpari, P; Shokoohi, Z; Behzadifar, Y. 2014. Energy use and carbon dioxide emission analysis in sugarcane farms: a survey on Haft-Tappeh Sugarcane AgroIndustrial Company in Iran. Journal of Cleaner Production (83):212-219.

Tormena, C; Araujo, M; Fidalsky, J; Imhoff, S; Da Silva A. 2008. Quantification of tensile strength and friability of an Oxisol (Acrudox) under no-tillage. Revista Brasilera de la Ciencia del Suelo 32(3):1-11.

USDA (United States Department of Agriculture, USA). 2010. Keys to Soil Taxonomy, Eleventh Ed. By Soil Survey Staff. Natural Resources Conservation Service. Washington, D.C. USA. p. 365.

Watts, C; Dexter, A. 1998. Soil friability: theory, measurement and the effects of management and organic carbon content. European Journal of Soil Science (49):73-84. 
